\title{
Repetitive Transcranial Magnetic Stimulation (rTMS) in Major Depression: Relation between Efficacy and Stimulation Intensity
}

Frank Padberg, M.D., Peter Zwanzger, M.D., Martin E. Keck, M.D., Norbert Kathmann, Ph.D., Patrick Mikhaiel, M.D., Robin Ella, M.D., Philipp Rupprecht, Heike Thoma, M.D., Harald Hampel, M.D., Nicola Toschi, Ph.D., and Hans-Jürgen Möller, M.D.

Repetitive transcranial magnetic stimulation (rTMS) has been found to exert modest to substantial antidepressant effects in the majority of prior clinical studies. As effect sizes and stimulation conditions have varied greatly, controversy persists regarding effective stimulation parameters (e.g. intensity, frequency, localization). In the present controlled study, we investigated whether the antidepressant efficacy of rTMS may be related to the stimulation intensity applied. Thirty-one patients suffering from a pharmacotherapy-resistant major depressive episode were randomly assigned to three treatment groups receiving rTMS at different stimulation intensities: (1) intensity at the individual motor threshold (MT); (2) 90\% subthreshold intensity; and (3) low intensity of standard sham rTMS. Each patient underwent 10 sessions of $10 \mathrm{~Hz}$ rTMS with
1500 stimuli/day over the left dorsolateral prefrontal cortex. Improvement of depressive symptoms after rTMS significantly increased with stimulation intensity across the three groups. A $30 \%$ to $33 \%$ reduction of baseline depression scores was observed after rTMS at MT intensity. Similarly, groups differed significantly regarding the clinical course after rTMS with the lowest number of antidepressant interventions and the shortest hospital stay in the MT intensity group. These findings support the hypothesis of a relationship between stimulation intensity of rTMS and its antidepressant efficacy.

[Neuropsychopharmacology 27:638-645, 2002] (C) 2002 American College of Neuropsychopharmacology. Published by Elsevier Science Inc.
KEY WORDS: Major depression; Medication resistance; Affective disorders; Transcranial magnetic stimulation; Stimulation intensity; Current density; Dorsolateral prefrontal cortex

From the Department of Psychiatry, Ludwig-Maximilian University (FP, PZ, NK, PM, RE, PR, HT, HH, H-JM), and Max Planck Institute of Psychiatry (MEK, NT), Munich, Germany

Address correspondence to: Frank Padberg, M.D., Department of Psychiatry, Ludwig-Maximilian University, Nussbaumstr. 7, 80336 Munich, Germany. Tel.: +01149-89-5160-5823; Fax: +01149-89-5160-

5322; E-mail: padberg@nk-i.med.uni-muenchen

Received February 28, 2001; revised November 19, 2001; accepted March 15, 2002.

Online publication: 3/25/02 at www.acnp.org/citations/Npp 032502270 .

This work is part of Philipp Rupprect's M.D. thesis at the LudwigMaximilian University.
Repetitive transcranial magnetic stimulation (rTMS) of the motor cortex has been widely used in neurophysiological studies of the human motor system (Hallett 1996). Motor evoked potentials (MEP) serve as experimental probes to study the impact of stimulation parameters (e.g. intensity or frequency) on motor functions. More recently, rTMS of the prefrontal cortex (PFC) was proposed to be a putative treatment in various psychiatric disorders: major depression, mania, posttraumatic stress disorder, obsessive compulsive disorder and schizophrenia (overview by George et al. 1999). Several controlled clinical trials have shown that rTMS exerts antidepressant effects superior to placebo (Pascual-Leone et al. 1996; George et al. 1997, 2000; Klein et al. 1999; Padberg et al. 1999; Berman et al. 2000; Garcia-Toro et al. 2001). However, only marginal effects 
were found in some studies (George et al. 1997; Padberg et al. 1999; Garcia-Toro et al. 2001) or no significant placeboverum differences could be detected (Loo et al. 1999; Manes et al. 2001). Moreover, several lines of preclinical evidence suggest that the molecular and cellular effects of chronic rTMS are similar to those observed following other forms of antidepressant therapy, namely drug treatment and electroconvulsive therapy (ECT) (Fleischmann et al. 1995; Zyss et al. 1997; Ben-Shachar et al. 1999; Keck et al. 2000, 2001).

Efforts to develop rTMS as a new antidepressant treatment in clinical practice are hampered, however, by the large variety of stimulation parameters used (e.g. intensity, frequency, train length, number of stimuli, stimulation site, and duration of treatment), and the lack of established parameter efficacy relationships (Padberg et al. 1999; George et al. 2000). One might expect that if rTMS exerts antidepressant action, efficacy would specifically depend on stimulation parameters, as it has been demonstrated for other antidepressant interventions, e.g. ECT (Sackeim et al. 2000). Here, we therefore tested the hypothesis that the antidepressant efficacy of rTMS is related to the stimulation intensity applied.

We report on a parallel design controlled study of 31 patients with a major depressive episode who were largely pharmacotherapy-resistant prior to rTMS. The patients were randomized into a two week trial of rTMS either at $100 \%$ motor threshold (MT) intensity, at 90\% MT intensity or at a low intensity sham condition, mathematically characterized by a series of computer-assisted, magnetic resonance imaging (MRI)-based reconstructions.

\section{METHODS AND MATERIALS}

\section{Patients}

Thirty-one inpatients from the Department of Psychiatry, Ludwig-Maximilian University Munich, partici- pated in this study. All patients were suffering from a moderate to severe major depressive episode. Diagnosis was established by experienced psychiatrists, based on a clinical interview following DSM-IV criteria. All patients provided their written informed consent for this study after the procedure had been fully explained. The ethics of the study were approved by the local institutional review board, and the study was carried out in accordance with the Declaration of Helsinki 1975. Patients with organic brain disorders or unstable medical conditions were excluded based on physical examination, routine laboratory analysis, electroencephalography and cranial computed tomography or magnetic resonance tomography. According to the safety criteria for rTMS (Wassermann 1998), patients with pacemakers, mobile metal implants or implanted medication pumps were also excluded. Table 1 summarizes demographic and clinical characteristics. All patients were deemed pharmacotherapy-resistant after administration of at least two antidepressant trials of adequate duration and dosage without significant clinical improvement (Helmchen 1990). One subject dropped out due to withdrawal of consent after the second rTMS session. The dosage of the current unsuccessful antidepressant treatment was kept constant for at least three weeks prior to rTMS. The pharmacological regime was not changed during rTMS therapy. Psychotropic medication was not tapered off in order to avoid confounding of results by pharmacological withdrawal effects or deterioration of clinical symptoms. Concomitant psychotropic medication was as follows (patient numbers in parentheses): tricyclics $(n=10)$, selective serotonin reuptake inhibitors $(\mathrm{n}=6)$, mono-amine oxidase inhibitors $(n=4)$, mirtazapine $(n=6)$, mianserin $(\mathrm{n}=1)$, maprotilin $(\mathrm{n}=2)$, venlafaxin $(\mathrm{n}=4)$, nefazodone $(n=2)$, reboxetine $(n=2)$, lithium $(n=8)$, buspirone $(n=1)$, neuroleptics $(n=12)$, benzodiazepines $(\mathrm{n}=10)$.

Table 1. Patient Characteristics (mean \pm SEM) at Baseline and F-values of Group Comparisons Using One-way ANOVAs (None of the F-values was Significant with 2/27 Degrees of Freedom).

\begin{tabular}{|c|c|c|c|c|}
\hline & $\begin{array}{c}100 \% \text { MT rTMS } \\
(\mathrm{n}=10)\end{array}$ & $\begin{array}{c}90 \% \text { MT rTMS } \\
\quad(n=10)\end{array}$ & $\begin{array}{l}\text { Sham rTMS } \\
\quad(n=10)\end{array}$ & $\mathbf{F}$ \\
\hline Age (yrs) & $62.1 \pm 4.6$ & $60.3 \pm 4.1$ & $52.7 \pm 5.7$ & 1.1 \\
\hline Gender (f/m) & $6 / 4$ & $7 / 3$ & $8 / 2$ & n.s. ${ }^{a}$ \\
\hline Previous episodes (No.) & $5.4 \pm 1.8$ & $3.9 \pm 0.9$ & $5.5 \pm 1.6$ & 0.4 \\
\hline Previous suicide attempts (No.) & $0.2 \pm 0.1$ & $0.5 \pm 0.3$ & $0.4 \pm 0.2$ & 0.6 \\
\hline Duration of current episode (months) & $17.1 \pm 7.7$ & $11.4 \pm 2.1$ & $7.9 \pm 1.7$ & 1.0 \\
\hline Antidepressant trials during episode (No.) & $4.8 \pm 0.8$ & $5.0 \pm 1.1$ & $5.7 \pm 1.1$ & 0.2 \\
\hline Baseline HRSD score & $23.6 \pm 1.9$ & $21.9 \pm 1.8$ & $24.4 \pm 2.1$ & 0.4 \\
\hline Baseline MADRS score & $28.7 \pm 2.0$ & $28.2 \pm 2.5$ & $30.4 \pm 2.0$ & 0.3 \\
\hline Motor threshold & $64 \pm 2.3$ & $61 \pm 3.7$ & $59 \pm 3.8$ & 0.5 \\
\hline
\end{tabular}

${ }^{a}$ not significant, $\chi^{2}=1.0$ 


\section{rTMS Methods}

A Magstim Rapid stimulator (The Magstim Company Ltd, Whitland, UK) was used with a focal $70 \mathrm{~mm}$ figure-eight shaped coil. On a separate day prior to the first treatment, the resting motor threshold (MT) for the right abductor pollicis brevis muscle (APBM) was determined with an Amplaid electromyograph (EMG) 14 (Fa. Micromed, Freiburg, Germany) according to the method of limits (Pascual-Leone et al. 1996). As in the majority of previous studies (George et al. 1995, 1997; Pascual-Leone et al. 1996; Berman et al. 2000; Grunhaus et al. 2000), the position of the left dorsolateral prefrontal cortex (DLPFC) was defined as $5 \mathrm{~cm}$ anterior to the scalp position for optimum stimulation of the right APBM in the parasagittal plane. This method for coil positioning has recently been reported to be moderately accurate in targeting DLPFC areas (Herwig et al. 2001). All patients underwent 10 afternoon sessions of rTMS at the left DLPFC within two weeks. Patients were randomly assigned to the following stimulation conditions: (1) $100 \%$ stimulation intensity related to MT; (2) $90 \%$ intensity related to MT; and (3) sham rTMS. For sham rTMS, the intensity was set at $100 \%$ MT and the coil was angled at $90^{\circ}$, one wing resting on the skull as in our earlier protocol (Padberg et al. 1999). Otherwise, stimulation parameters were identical for the three conditions: 1500 stimuli/day, $10 \mathrm{~Hz}, 10 \mathrm{~s}, 15$ trains, $30 \mathrm{~s}$ intertrain-interval.

The patients were naive to rTMS prior to the study and not familiar with the differences between sham and verum rTMS regarding its acoustic and tactile artifacts.

\section{Partial Model of Induced Current Density}

The current density distribution in the brain was modeled for all rTMS conditions and is shown for 100\% MT and sham rTMS (Figure 1). Calculations were carried out as previously reported (Keck et al. 2000, 2001). Briefly, the electrical characteristics of the brain were reconstructed from a complete set of MRI images by mapping the conductivity distribution of the tissue onto the anatomical data. This map consists of a fourth degree interpolating polynomial using the grayscale values (0-255) of the MRI images as the argument and returning the corresponding conductivity value at that point in space. The five known data points used to interpolate are taken from the literature (Cerri et al. 1995), values ranging from $0.01 \mathrm{~A} / \mathrm{Vm}$ (bone) to $1.6 \mathrm{~A} / \mathrm{Vm}$ (cerebrospinal fluid). Then, the magnetic induction field is calculated as function of coil geometry plus the time evolution of the incoming current with $64 \%$ of the maximal output of the stimulator (average MT). Thereafter, discretized versions of Maxwell's equations in integral form are solved simultaneously for all meshes in the network (and hence for the domain of interest). This allows for exact character- ization of the total electric field and current density distributions arising in the brain. The peak current densities were related as follows: $100 \%: 90 \%: 40 \%$ over the three conditions (100\% MT: $90 \%$ MT: sham). The ratios of activated volumes between sham and 100\% MT were defined as function of a virtual threshold ( 5 to $100 \mathrm{~A} / \mathrm{m}^{2}$ ) ranging from 0.48 to 0.01 .

\section{Clinical Assessment}

Patients were examined by a psychiatrist uninvolved in rTMS treatment and blinded to the rTMS condition. Severity of depression was assessed using the 21-item version of the Hamilton Rating Scale for Depression (HRSD) (Hamilton 1960) and the Montgomery-Asberg Depression Rating Scale (MADRS) (Montgomery and Asberg 1979) at baseline, as well as after one and two weeks of rTMS. A 7-point clinical global impression (CGI) of severity scale was used as overall outcome measure. Side effects, tolerability and rTMS-induced sensations were documented daily by the use of brief questionnaires and visual analog scales (VAS). After discharge from the hospital, patients' charts were evaluated retrospectively by a research assistant blinded to the treatment conditions. Information regarding duration of the hospital stay and number of antidepressant trials was obtained for all patients.

\section{Statistical Methods}

The results are expressed as means \pm SEM. All statistical tests were 2-tailed and the level of significance was chosen at $p<.05$. One-way analysis of variance (ANOVA) was used as a parametric approach to test for inter-group differences in demographic and pretreatment clinical variables. Repeated-measures ANOVAs were applied for the analysis of rTMS efficacy, with treatment group (three levels) as the between-subjects factor and time point (baseline, week 1, week 2) as the within-subjects factor. Pearson's correlation coefficients were calculated to test whether there was an association between antidepressant efficacy and stimulation intensity related to the output of the stimulator. For the sham condition individual intensities were multiplied by the factor 0.4, which was identified in the mathematical model to characterize the relation between induced current densities of condition 1 and 3 (100\% MT sham coil position and $100 \%$ MT verum). Frequencies of responders in verum and sham cells were compared using a chi-square test. One-way ANOVAs were performed to evaluate clinical variables assessed between termination of rTMS and discharge of patients. Bonferroni posthoc tests were used to determine which pairs of groups were statistically different. Analyses were performed using SPSS Version 10.0. 


\section{$100 \% \mathrm{MT}$}

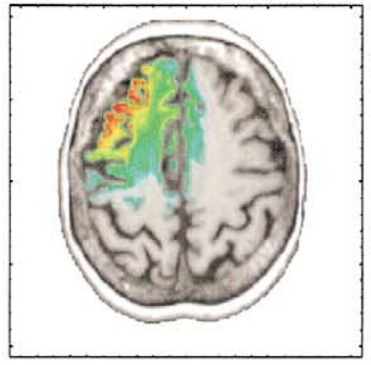

$-1 \mathrm{~cm}$

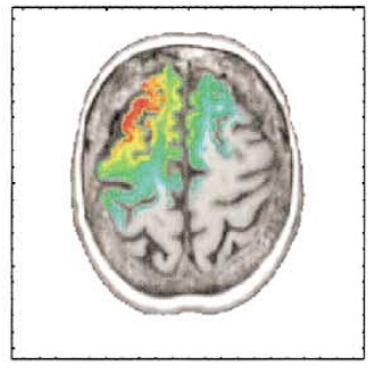

$0 \mathrm{~cm}$

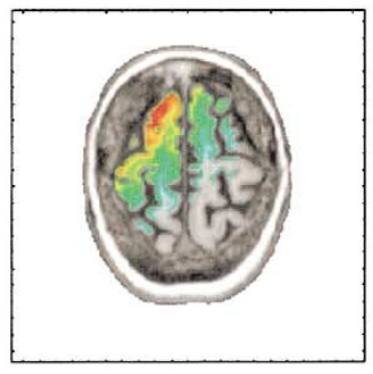

$+1 \mathrm{~cm}$

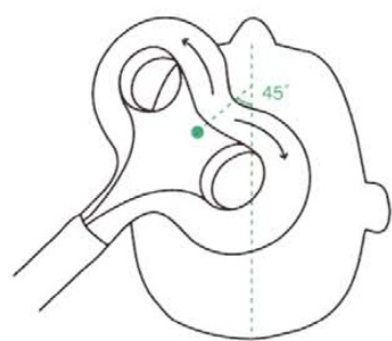

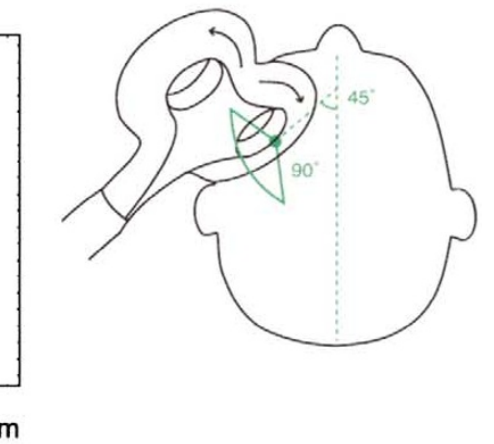

\section{sham rTMS}
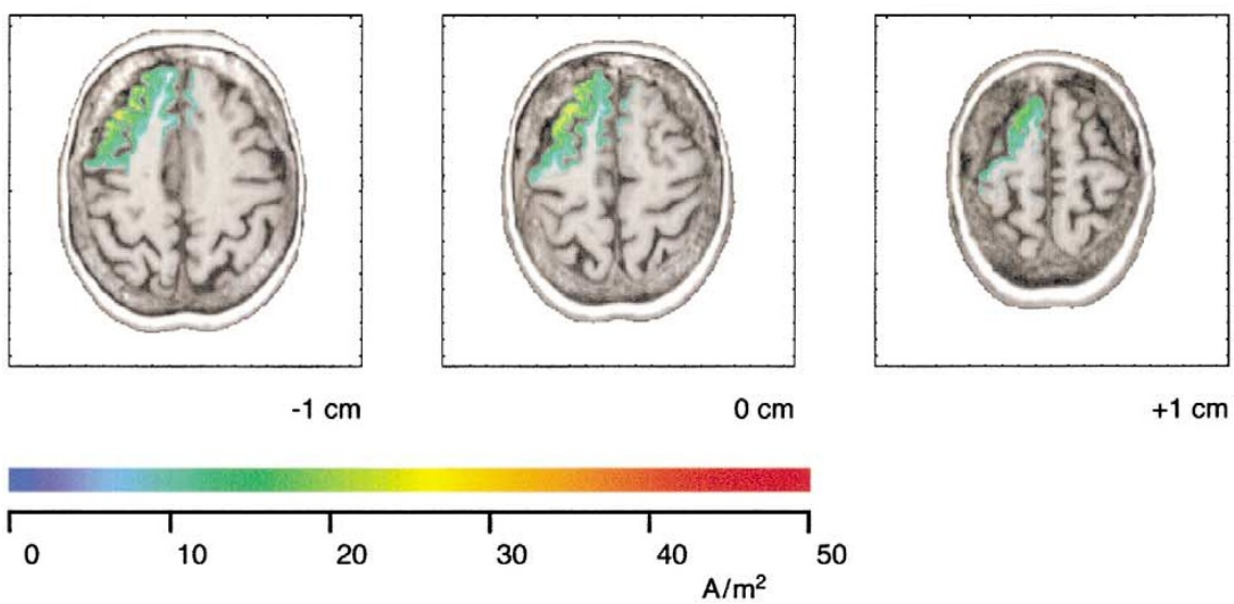

Figure 1. MRI-based partial model of the induced current density for verum and sham rTMS conditions: $100 \%$ MT intensity with normal coil position and sham coil position (also 100\% MT intensity). Transverse MRI sections are shown at the level of the coil touching the skull, as well as $1 \mathrm{~cm}$ below and $1 \mathrm{~cm}$ above this level. Coil orientations are indicated.

\section{RESULTS}

\section{Group Characteristics}

Baseline characteristics of treatment groups are shown in Table 1. No significant group differences for demographic data were found. On average, patients had 4.9 $( \pm 0.9)$ previous depressive episodes with a mean duration of $12( \pm 2.7)$ months of the current episode. They were resistant to a mean of $5.2( \pm 0.6)$ adequate antidepressant trials. There were no significant differences between treatment groups either by means of the number of medication trials during the current episode or by the duration of the current episode. No significant difference was found between groups for baseline HRSD, MADRS or CGI scores. There were no significant group differences in the frequencies of antidepressants including their subclasses, of tranquilizers and of neuroleptics during rTMS treatment (not shown).

\section{Clinical Efficacy}

Across treatment groups, depression scores significantly declined during rTMS (MADRS: $\mathrm{F}_{2,54}=13.0 ; p<$
.001 ; HRSD: $\left.\mathrm{F}_{2,54}=8.9 ; p<.001\right)$. The main effect of treatment group was not significant (MADRS: $\mathrm{F}_{2,27}=$ 1.6; n.s.; HRSD: $F_{2,27}=1.2$; n.s.). Interaction of treatment group with time was significant for MADRS scores $\left(\mathrm{F}_{4,54}=2.9 ; p<.05\right)$ but not for HRSD scores $\left(\mathrm{F}_{4,54}=1.4\right.$.; n.s.). To resolve differential treatment effects, the differences between baseline and week 2 scores were computed and 1-way ANOVAs testing polynomial contrasts were performed. For MADRS difference scores, the linear component of this contrast was significant $(p<$ $.05)$, whereas the quadratic component was not. This means that the antidepressant effect linearly increased from sham rTMS to $90 \%$ MT rTMS and to $100 \%$ MT rTMS. Expressed in percent decrease of MADRS scores, sham rTMS yielded a $4.1 \%$ ( \pm 5.2 ) reduction, $90 \%$ MT rTMS resulted in a $15.1 \%( \pm 6.6)$ decrease, and $100 \%$ MT rTMS reduced MADRS scores by $33.2 \%$ ( \pm 8.9$)$. The respective linear effect on HRSD scores showed a statistical trend $(p<.10)$. Percent reductions of HRSD scores were $7.1 \%( \pm 5.8)$ after sham rTMS, $14.9 \%( \pm 8.9)$ after 90\% MT rTMS and 29.6\% ( \pm 8.7) after 100\% MT rTMS. Mean depression scores at the three measurement points are shown in Figure 2. CGI scores paralleled the 


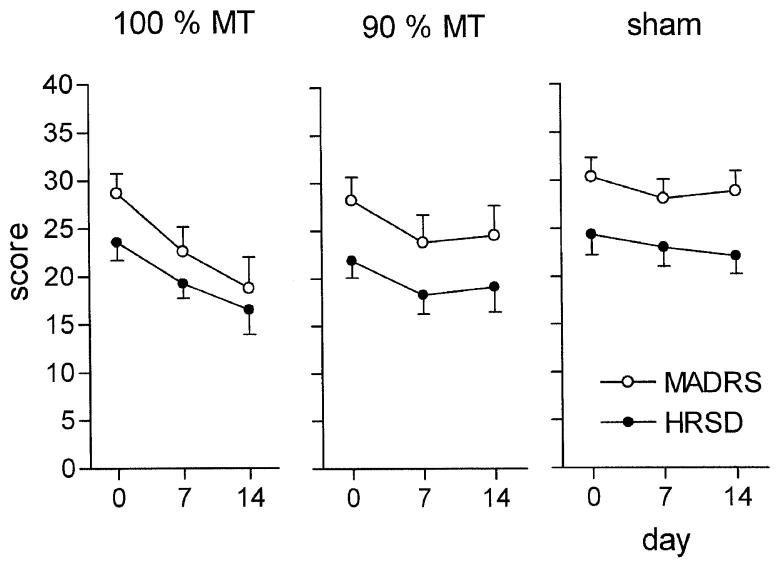

Figure 2. Effects of $100 \%$ MT intensity, 90\% MT intensity and sham rTMS on mean scores ( \pm SEM) of the Hamilton Rating Scale for Depression (HRSD) and the Montgomery-Asberg Depression Rating Scale (MADRS) before rTMS, and at day 7 and day 14 of the study. For MADRS scores, the antidepressant effect significantly increased from sham rTMS over $90 \%$ MT rTMS to $100 \%$ MT rTMS $(p<.05)$. The respective linear effect for HRSD scores showed only a trend $(p<.10)$.

effects of depression scores (not shown). For CGI scores, the effect of time (across groups) and the interaction of time and treatment group were significant $\left(\mathrm{F}_{2,54}=\right.$ $5.7 ; p<.01$ and $\left.\mathrm{F}_{2,54}=3.8 ; p<.05\right)$.

More patients tended to respond in verum cells $(100 \%$ and $90 \% \mathrm{MT})$ compared with the sham group $\left(\chi^{2}=3.0\right.$; $p=.08)$ according to classical response criteria $(\geqslant 50 \%$ HRSD score reduction from baseline). Five of 20 patients responded in verum cells, but none in the sham group. In the $100 \%$ MT group, two remitters (HRSD score $<9$ ), three responders and two partial responders $(50 \%>$ HRSD score reduction $\geqslant 25 \%$ ) were found after rTMS treatment (Table 2). After $90 \%$ MT rTMS, one remitter, two responders and no partial responders were detected. Following sham rTMS, only two partial responders were found.

Duration of the hospital stay after rTMS treatment and number of antidepressant trials until discharge from hospital were retrospectively assessed based on the patients' charts (Table 2). One-way ANOVAs showed statistically significant differences between groups for both duration of the hospital stay $\left(\mathrm{F}_{2,27}=4.2 ; p<.05\right)$ and number of required antidepressant trials $\left(\mathrm{F}_{2,27}=\right.$ 10.2; $p<.001$ ). Post-hoc comparisons (Bonferroni) showed that the number of antidepressant medication trials was significantly higher and duration of the hospital stay after rTMS was longer in the sham rTMS group than in the $90 \%$ and $100 \%$ MT rTMS groups.

To address the question whether there is a correlation between efficacy and stimulation intensity expressed as percent maximal output of the stimulator, we analyzed this correlation across all subjects by correcting for the less effective coil position in the sham group. A negative correlation between the stimulation intensity related to the output of the stimulator and the reduction of MADRS scores (Figure 3) was found ( $r=$ $-0.42 ; p<.05)$. The respective correlation was not significant for HRSD scores ( $r=-0.3$; n.s.).

\section{Side Effects and Tolerability}

No severe side effects of rTMS were observed. Five patients reported an aversive tactile artifact during rTMS (two patients with $100 \%$, three with $90 \%$ and none with sham rTMS) and five patients experienced the rTMS as generally unpleasant (two patients with 100\%, three with $90 \%$ and none with sham rTMS). Mild headaches and numbness of the left temple were reported by two patients. In another patient, a migraine attack occurred within four hours after sham rTMS. VAS rating of pain during rTMS showed no general perceptual change of pain intensity over the two weeks. No spread of excitation monitored by EMG and no seizures occurred.

\section{DISCUSSION}

In this controlled parallel design study three different fast rTMS conditions were compared as treatment in largely pharmacotherapy-resistant patients. Clinical improvement after rTMS significantly increased across the three groups, with best results at motor threshold (MT) intensity, little improvement after subthreshold stimulation and no improvement after low intensity stimulation. We regard this finding as a first and preliminary

Table 2. Number of Remitters and Responders, Required Antidepressant Trials, and Duration of the Hospital Stay after 100\% MT Intensity, 90\% MT Intensity and Sham rTMS (mean \pm SEM).

\begin{tabular}{lccc}
\hline & $\begin{array}{c}\mathbf{1 0 0 \%} \text { MT rTMS } \\
(\mathbf{n}=\mathbf{1 0})\end{array}$ & $\begin{array}{c}\mathbf{9 0 \%} \text { MT rTMS } \\
(\mathbf{n}=\mathbf{1 0})\end{array}$ & $\begin{array}{c}\text { Sham rTMS } \\
(\mathbf{n}=\mathbf{1 0})\end{array}$ \\
\hline Remission $^{\mathrm{a}}$ (No.) & 2 & 1 & 0 \\
Response (R/PR/NR) $^{\mathrm{b}}$ (No.) & $3 / 2 / 5$ & $2 / 1 / 7$ & $0 / 2 / 8$ \\
Antidepressant trials after rTMS (No.) $_{\text {Duration of the hospital stay after rTMS (days) }}$ & $1.4 \pm 0.2$ & $1.6 \pm 0.2$ & $3.6 \pm 0.6$ \\
\hline
\end{tabular}

${ }^{\text {a }}$ defined as HRSD score $<9$ after rTMS

${ }^{\mathrm{b}} \mathrm{R}$ - responder (HRSD reduction after $\left.\mathrm{rTMS} \geqslant 50 \%\right), \mathrm{PR}-$ partial responder $(50 \%>$ HRSD reduction $\geqslant 25 \%), \mathrm{NR}-\mathrm{non}-\mathrm{responder}(\mathrm{HRSD}$ reduction $<25 \%$ ). 


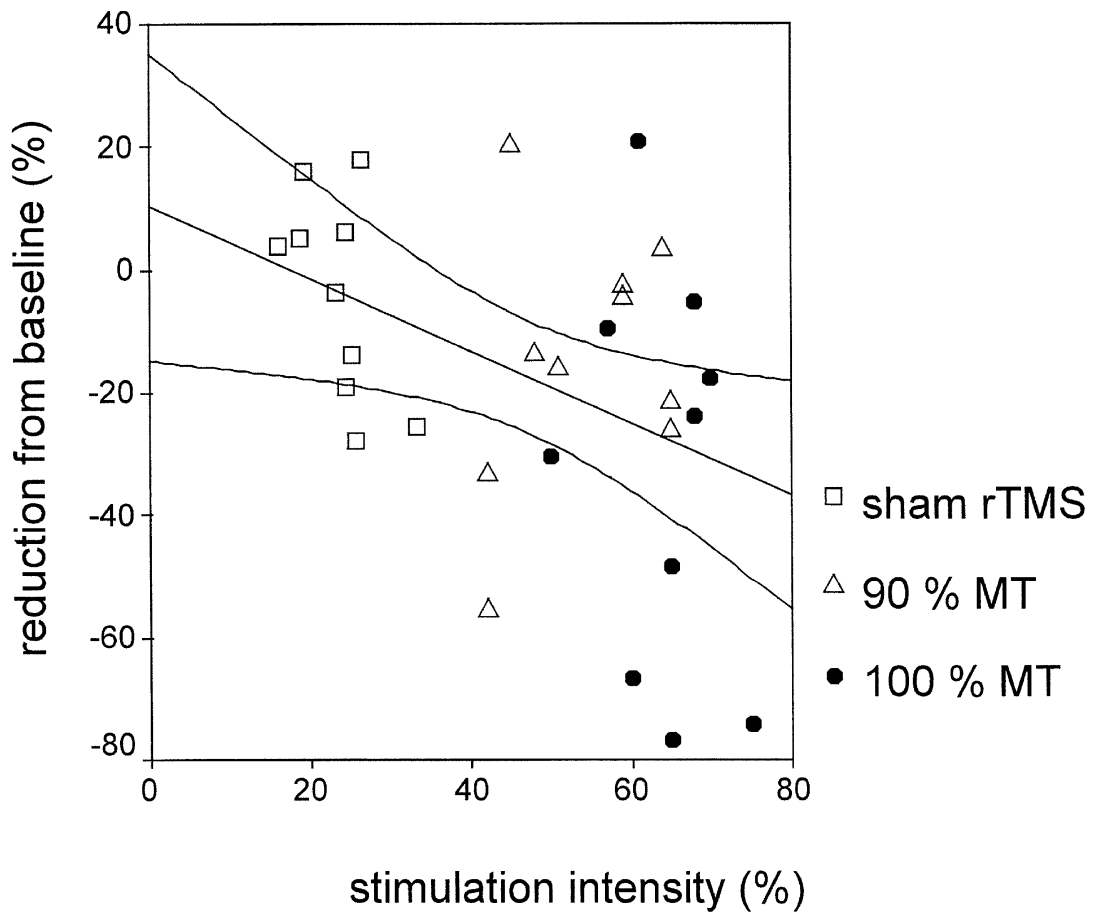

Figure 3. Correlation between reduction of MADRS scores from baseline and intensity related to the stimulation intensity (\% output of the stimulator). Based on the partial model, the individually applied output-related intensities for sham rTMS were corrected by 0.4 to consider the less effective coil position. The regression line including the $95 \%$ confidence interval is indicated.

evidence of a relation between antidepressant efficacy and stimulation intensity.

Antidepressant effects of fast rTMS of the left DLPFC have been demonstrated in the majority of previous controlled studies (Pascual-Leone et al. 1996; George et al. 1997, 2000; Klein et al. 1999; Padberg et al. 1999; Berman et al. 2000; Garcia-Toro et al. 2001), although some investigators found only marginal or no significant effects, when compared with sham rTMS (George et al. 1997; Padberg et al. 1999; Garcia-Toro et al. 2001; Loo et al. 1999, Manes et al. 2001). Despite the range of stimulation intensities in prior studies, solid conclusions regarding the relationship between efficacy and intensity were impeded by: (1) the lack of a direct comparison between different intensities; and (2) the problem that studies widely varied with respect to other stimulation parameters (frequency, number of stimuli, etc.) and clinical characteristics of patient samples. Two placebo-controlled cross-over trials suggested an antidepressant efficacy of rTMS at $80-90 \%$ MT (Pascual-Leone et al. 1996; George et al. 1997) with decreases in HRSD scores ranging from $17 \%$ (George et al. 1997) to $45 \%$ (PascualLeone et al. 1996). Similarly, parallel design sham controlled studies showed HRSD score reductions between $17 \%$ and $26 \%$ intensity with a stimulation intensity of $90 \%$ MT (Padberg et al. 1999; Garcia-Toro et al. 2001) and of $35 \%$ with $100 \%$ MT intensity (George et al. 2000). In an- other trial, a $38 \%$ reduction of the HRSD score was reported after rTMS at only $80 \%$ MT. However, MT was visually detected, which presumably led to a slightly higher MT than in studies where MT was determined using EMG (Berman et al. 2000).

In the present study, we observed a HRSD decrease by $30 \%$ after $100 \%$ MT rTMS and by $15 \%$ after $90 \%$ MT rTMS in patients who had previously been pharmacotherapyresistant to five antidepressant trials on average. Moreover, depression and CGI scores revealed statistically significant differences between groups whereby a linear increase of efficacy was shown with maximal effects after $100 \%$ MT rTMS. Thus, the effect sizes found in the present study are within the range observed in previous studies and appear to be smaller than antidepressant effects observed after ECT (Sackeim et al. 2000; McCall et al. 2000).

The main finding of this study was that antidepressant efficacy increased in an intensity-related fashion over treatment groups. An intensity efficacy relationship would be consistent with previous findings: (1) There is an obvious relationship between the MEP amplitude and the intensity of single pulse TMS (input-output curve) (Hallett 1996); MEP amplitudes also increase during a train of $5 \mathrm{~Hz}$ rTMS in an intensity-dependent manner, however, effects of rTMS on MEP amplitudes are complex and vary with rTMS frequency (Pascual-Leone et al. 1994); (2) Effects of rTMS on non-motor tasks are inten- 
sity dependent (e.g. induction of speech arrest, suppression of phosphene generation) (Jennum et al. 1994; Boroojerdi et al. 2000); (3) Using interleaved fMRI and rTMS, an intensity-dependent increase of the BOLD signal was reported during rTMS of the motor cortex (Bohning et al. 1999) as well as of the left prefrontal cortex (Nahas et al. 2001).

The sham condition with the coil angled at $90^{\circ}$, one wing resting on the skull, was mathematically characterized as inducing a low peak current density in the brain ( $40 \%$ in relation to the normal coil position). The partial model applied had originally been established in order to compare stimulation effects between humans and animals (Keck et al. 2000, 2001). The assumption of a weak active sham condition is in line with previous experimental evidence in monkeys and humans, showing that some sham conditions are capable of electrically stimulating the brain, depending on the respective coil position (Loo et al. 2000; Lisanby et al. 2001). Compared with these experimental approaches, our mathematical model is simplified and does not consider all relevant issues; for example, the precise and individual impact of cortical cytoarchitecture and folding on the induced currents in the cortex. However, modeling neurophysiological effects of rTMS where experimental measures are not accessible may be a promising approach to further characterize applied treatment conditions and generate new modalities and applications for rTMS, particularly in conjunction with functional neuroimaging. Moreover, to define stimulation conditions more accurately in rTMS treatment studies is particularly important, since it cannot be excluded that weak active sham conditions obscure effects of the compared verum conditions particularly in studies with small sample sizes (Loo et al. 1999, 2000). However, in the study of Loo and colleagues, alternative explanations for the large effect in the sham treated group have to be considered; for example, permissive definition of therapy-resistance, and concomitant psychotropic medication with stable antidepressant medication for only two weeks prior to rTMS.

The present study does not allow an answer to the question whether rTMS intensity related to the individual MT or intensity related to the maximal output of the stimulator is more relevant for the observed dose efficacy relationship, as both measures are linked and patients were grouped by MT intensity. However, it is not obvious that MT, which is a genuine measure of excitability in a cortical network producing the volley in the corticospinal tract (Hallett 1996), is useful for other cortical areas to individually adjust stimulation intensity, though MT is commonly used to restrict stimulation parameters with respect to rTMS safety (Wassermann 1998). A similar problem was very recently solved for right unilateral ECT using a fixed dose that was not adjusted to the individual seizure threshold (ST): the stim- ulus dose relative to the ST was shown to be the most significant predictor of antidepressant response (McCall et al. 2000). An analogous approach is warranted in future rTMS studies addressing this issue.

The interpretation of our findings is limited by the small sample size and the comparison of only three conditions, none of which exceeded $100 \%$ MT intensity for safety concerns. Therefore, we regard our findings as preliminary. As the hypothesized relationship between intensity and efficacy has major implications for the therapeutic use of rTMS, further studies are needed to prove this hypothesis and investigate rTMS over a larger range of MT-related intensities.

\section{ACKNOWLEDGMENTS}

The authors wish to thank Dr. Florian Müller-Siecheneder, Dr. Thomas Frodl, Dr. Elisabeth Hofschuster and Dr. Ulrich W. Preuss for the clinical rating. They further thank Mrs. J. Klesing and Dr. Martin Strassnig for critically reading the manuscript. This project was supported by the German Federal Research Ministry within the promotional emphasis "Competence Nets in Medicine" (Subproject 4.5, M.E.K., Subproject 6.5, F.P.).

\section{REFERENCES}

Ben-Shachar D, Gazawi H, Riboyad-Levin J, Klein E (1999): Chronic repetitive transcranial magnetic stimulation alters beta-adrenergic and 5-HT2 receptor characteristics in rat brain. Brain Res 816:78-83

Berman RM, Narasimhan M, Sanacora G, Miano AP, Hoffman RE, Hu XS, Charney DS, Boutros NN (2000): A randomized clinical trial of repetitive transcranial magnetic stimulation in the treatment of major depression. Biol Psychiatry 47:332-337

Bohning DE, Shastri A, McConnell KA, Nahas Z, Lorberbaum JP, Roberts DR, Teneback C, Vincent DJ, George MS (1999): A combined TMS/fMRI study of intensitydependent TMS over motor cortex. Biol Psychiatry 45:385-394

Boroojerdi B, Prager A, Muellbacher W, Cohen LG (2000): Reduction of human visual cortex excitability using $1-\mathrm{Hz}$ transcranial magnetic stimulation. Neurology 54: 1529-1531

Cerri G, De Leo R, Moglie F, Schiavoni A (1995): An Accurate 3-D model for magnetic stimulation of the brain cortex. J Med Eng Tech 19:7-16

Fleischmann A, Prolov K, Abarbanel J, Belmaker RH (1995): The effect of transcranial magnetic stimulation of rat brain on behavioral models of depression. Brain Res 699:130-132

Garcia-Toro M, Mayol A, Arnillas H, Capllonch I, Ibarra O, Crespi M, Mico J, Lafau O, Lafuente L (2001): Modest adjunctive benefit with transcranial magnetic stimulation in medication-resistant depression. J Affect Disord 64:271-275

George MS, Wassermann EM, Williams WA, Callahan A, 
Ketter TA, Basser P, Hallett M, Post RM (1995): Daily left prefrontal repetitive transcranial magnetic stimulation (rTMS) improves mood in depression. Neuroreport 6:1853-1856

George MS, Wassermann EM, Kimbrell TA, Little JT, Williams WE, Danielson AL, Greenberg BD, Hallett M, Post RM (1997): Mood improvement following daily left prefrontal repetitive transcranial magnetic stimulation in patients with depression: A placebo-controlled crossover trial. Am J Psychiatry 154:1752-1756

George MS, Lisanby SH, Sackeim HA (1999): Transcranial magnetic stimulation. Arch Gen Psychiatry 56:300-311

George MS, Nahas Z, Molloy M, Speer AM, Oliver NC, Li X-B, Arana GW, Risch SC, Ballenger JC (2000): A controlled trial of daily left prefrontal cortex TMS for treating depression. Biol Psychiatry 48:962-970

Grunhaus L, Dannon PN, Schreiber S, Dolberg OH, Amiaz R, Ziv R, Lefkifker E (2000): Repetitive transcranial magnetic stimulation is as effective as electroconvulsive therapy in the treatment of nondelusional major depressive disorder: an open study. Biol Psychiatry 47:314-324

Hallett M (1996): Transcranial magnetic stimulation: a tool for mapping the central nervous system. Electroencephalogr Clin Neurophysiol (Suppl 46):43-51

Hamilton M (1960): A rating scale for depression. J Neurol Neurosurg Psychiatry 23:56-62

Helmchen H (1990): Gestuftes Vorgehen bei Resistenz gegen Antidepressiva-Therapie. In Möller HJ (ed), Therapieresistenz unter Antidepressivabehandlung. New York, Springer, pp 237-250

Herwig U, Padberg F, Unger J, Spitzer M, SchonfeldtLecuona C (2001): Transcranial magnetic stimulation in therapy studies: examination of the reliability of "standard" coil positioning by neuronavigation. Biol Psychiatry 50:58-61

Jennum P, Friberg L, Fuglsang-Frederiksen A, Dam M (1994): Speech localization using repetitive transcranial magnetic stimulation. Neurology 44:269-273

Keck ME, Engelmann M, Müller MB, Henniger MSH, Hermann B, Rupprecht R, Neumann ID, Toschi N, Landgraf R, Post A (2000): Repetitive transcranial magnetic stimulation induces active coping strategies and attenuates the neuroendocrine stress response in rats. J Psychiatr $\operatorname{Res} 34: 265-276$

Keck ME, Welt T, Post A, Müller MB, Toschi N, Wigger A, Landgraf R, Holsboer F, Engelmann M (2001): Neuroendocrine and behavioral effects of repetitive transcranial magnetic stimulation in a psychopathological animal model are suggestive of antidepressant-like effects. Neuropsychopharmacology 24:337-349

Kimbrell TA, Little JT, Dunn RT, Frye MA, Greenberg BD, Wassermann EM, Repella JD, Danielson AL, Willis MW, Benson BE, Speer AM, Osuch E, George MS, Post RM (1999): Frequency dependence of antidepressant response to left prefrontal repetitive transcranial magnetic stimulation (rTMS) as a function of baseline cerebral glucose metabolism [In Process Citation]. Biol Psychiatry 46:1603-1613

Klein E, Kreinin I, Chistyakov A, Koren D, Mecz L, Marmur S, Ben-Shachar D, Feinsod M (1999): Therapeutic effi- cacy of right prefrontal slow repetitive transcranial magnetic stimulation in major depression. Arch Gen Psychiatry 56:315-320

Lisanby SH, Gutman D, Luber B, Schroeder C, Sackeim HA (2001): Sham TMS: intracerebral measurement of the induced electrical field. Biol Psychiatry 49:460-463

Loo C, Mitchell P, Sachdev P, McDarmont B, Parker G, Gandevia S (1999): Double-blind controlled investigation of transcranial magnetic stimulation for the treatment of resistant major depression. Am J Psychiatry 156:946-948

Loo CK, Taylor JL, Gandevia SC, McDarmont BN, Mitchell PB, Sachdev PS (2000): Transcranial magnetic stimulation (TMS) in controlled treatment studies: are some "sham" forms active? Biol Psychiatry 47:325-331

Manes F, Jorge R, Morcuende M, Yamada T, Paradiso S, Robinson RG (2001): A controlled study of repetitive transcranial magnetic stimulation as a treatment of depression in the elderly. Int Psychogeriatr 13:225-231

McCall W, Reboussin DM, Weiner RD, Sackeim HA (2000): Titrated moderately suprathreshold vs fixed high-dose right unilateral electroconvulsive therapy. Arch Gen Psychiatry 57:438-444

Montgomery S, Asberg M (1979): A new depression scale designed to be sensitive to change. Br J Psychiatry 134:382-389

Nahas Z, Lomarev M, Roberts DR, Shastri A, Lorberbaum JP, Teneback CC, McConnell KA, Vincent DJ, Li X, George MS, Bohning DE (2001): Transcranial magnetic stimulation (TMS) produces intensity-dependent bilateral effects as measured by interleaved BOLD fMRI. Biol Psychiatry (in press)

Padberg F, Zwanzger P, Thoma H, Kathmann N, Haag C, Greenberg BD, Hampel H, Möller HJ (1999): Repetitive transcranial magnetic stimulation (rTMS) in pharmacotherapy-refractory major depression: comparative study of fast, slow and sham rTMS. Psychiatry Res 88:163-171

Pascual-Leone A, Valls-Solé J, Wassermann EM, Hallett M (1994): Responses to rapid-rate transcranial magnetic stimulation of the human motor cortex. Brain 117:847-858

Pascual-Leone A, Rubio B, Pallardó F, Catalá MD (1996): Rapid-rate transcranial magnetic stimulation of left dorsolateral prefrontal cortex in drug-resistant depression. Lancet 348:233-237

Sackeim HA, Prudic J, Devanand DP, Nobler MS, Lisanby SH, Peyser S, Fitzsimons L, Moody BJ, Clark J (2000): A prospective, randomized, double-blind comparison of bilateral and right unilateral electroconvulsive therapy at different stimulation intensities. Arch Gen Psychiatry 57:425-434

Wassermann EM (1998): Risk and safety of repetitive transcranial magnetic stimulation: report and suggested guidelines from the International Workshop on the Safety of Repetitive Transcranial Magnetic Stimulation, June 5-7, 1996. Electroenceph Clin Neurophysiol 998:1-16

Zyss T, Gorka Z, Kowalska M, Vetulani J (1997): Preliminary comparison of behavioral and biochemical effects of chronic transcranial magnetic stimulation and electroconvulsive shock in the rat. Biol Psychiatry 42:920-924 